\title{
Stability Analysis of a Proximate Time Optimal Controlled Electrostatic Suspension System Using Piezo Actuator via Lyapunov Functional Approach
}

\author{
T. T. Le \\ Department of Mechanical Engineering, Ho Chi Minh City University of Food Industry, Ho Chi Minh City, Vietnam \\ Correspondence should be addressed to T. T. Le; truyenlt@hcmut.edu.vn
}

Received 13 July 2019; Revised 13 September 2019; Accepted 8 October 2019; Published 22 January 2020

Academic Editor: Krzysztof Kecik

Copyright @ 2020 T. T. Le. This is an open access article distributed under the Creative Commons Attribution License, which permits unrestricted use, distribution, and reproduction in any medium, provided the original work is properly cited.

\begin{abstract}
An electrostatic suspension of silicon wafer using proximate time optimal control has been successfully developed. In this system, the movable electrodes which are supplied by constant voltage and actuated by the piezoelectric (PZT) actuator are used instead of stationary electrodes like previous systems. The changing of the gap length between movable electrodes and the suspended object will create varying capacitances that can control the electrostatic forces. To overcome the problem of actuator saturation of the piezo actuator, a proximate time optimal control is used to stabilize the system. The system stability is theoretically investigated using Lyapunov's function. The constant voltage supplying to the electrode is an important parameter, and it is also determined. The paper presents series of the simulation and experimental results that prove completely suspension of 4 -inch silicon wafer without any mechanical contact.
\end{abstract}

\section{Introduction}

The electrostatic suspension has several advantages compared to electromagnetic levitation since they can suspend various materials such as conductors, semiconductors, and dielectric materials [1-4]. Another field where electrostatic suspension systems could be applied is the processing of materials without any contact with containers, crucibles, or walls. This method allows the processing of corrosive materials or materials whose melting temperatures are higher than that of the crucible. Handling of high-temperature materials in their solid or liquid phases is thus achievable. The physicochemical contamination from the crucible or walls can be avoided because of no physical contact with a container, and the thermodynamic property determination of the original material can be obtained [5].

In general, the electrostatic suspension systems utilize high-voltage amplifier to generate high voltage to suspend objects as silicon wafer, hard disk media, and glass panels [6-11]. However, the use of the expensive and bulky highvoltage amplifiers is the disadvantage especially in case the suspension of large flexible objects [12].
The cost-effective electrostatic suspension system based on switched voltage control scheme has been studied and reported $[13,14]$. Instead of using high-voltage amplifiers, these systems use a single high-voltage power that can supply a constant voltage for an arbitrary number of individual stator electrodes that have to be controlled. However, the vibration of suspended object could not disappear due to existent delay times which are always exist in the real systems having switching circuits. In addition, the amplitude of vibration of object is proportional of time delays. This reason can be attributed an unstable of the suspension systems [15].

It is known that the electrostatic force is inversely proportional to the square of airgap length. By controlling the gap so as to balance the gravity and the electrostatic force, the object such as silicon wafer can be actively levitated. A new method to perform contactless suspension by using electrostatic force is proposed to overcome the disadvantages in previous systems that are bulky and expensive and have big amplitudes of vibration.

Firstly, in order to remove the effect of time delay explained above, a simple way is that the switching circuit is not employed. A single high-voltage supply is utilized to 
provide directly constant voltages to movable electrodes for generation of an electrostatic force. Secondly, a piezoelectric actuator with rapid deformation characteristic is employed to generate movements of the movable electrodes to change the gap length between movable electrodes and suspended object. As a result of that, the electrostatic forces could be controlled by varying capacitance formed between them. The piezo actuator is chosen to generate movements of the movable electrodes because of its simple construction and low potential for thermal problems. The piezoelectric actuator can move linearly with the nanometer displacement resolution, it is widely used in micro-/nano-positioning $[16,17]$ and optics $[18,19]$. However, the stacked piezoelectric actuator often has the travel stroke limitation of about one hundred microns. To enlarge the displacement of PZT actuator, a lever type displacement amplifying mechanism is used in this work.

Typically, due to the PZT actuator limited travel range, the control input should be constrained to avoid damage to the PZT ceramics. When the actuator is saturated, the performance of the control system designed will seriously deteriorate [20]. Therefore, the controllers are designed by constraining the control input not to hit the saturation limit.

The ideal time optimal controllers (TOC) that are bangbang type are not good in practice, because they are sensitive to disturbance, parameter variations, and unmodeled dynamic. In any real control system, there will be process and measurement noise, uncertainty in the system parameters, and finite actuation bandwidth which will cause the control to chatter between its maximum and minimum values; thus, TOC is not practical in real applications [20-23]. To overcome the chattering problem in TOC caused by measurement noises, feedback delays, and model uncertainties, a modified technique under the name the continuous proximate time optimal control is applied in our electrostatic suspension system, and stability of the system was investigated. As the system output approaches the reference point, the controller switches to a linear control law, thus eliminating chattering and providing feedback in order to accommodate plant uncertainty and measurement noise. Furthermore, a limitation of the piezo actuator displacement is taken into account.

This paper is organized as follows: in Sections 2 and 3, we present the principle of operation and the dynamic motion of the suspended object. The design of the proximate time optimal control for the suspension system and the stability analysis are presented in Section 4. The experimental results are presented in Section 5. Finally, the conclusion is given in Section 6.

\section{Principle of Operation}

The schematic of a 1-DOF electrostatic suspension using piezoelectric actuator is presented in Figure 1 where the system consists of a suspended object is 4 -inch silicon wafer, fixed airgap sensor, two concentric movable electrodes $E_{\mathrm{n}}$ and $E_{\mathrm{p}}$ which are supplied by constant voltage by one single high-voltage supply, a piezoelectric actuator to generate a movement of the movable electrodes, a controller, a drive

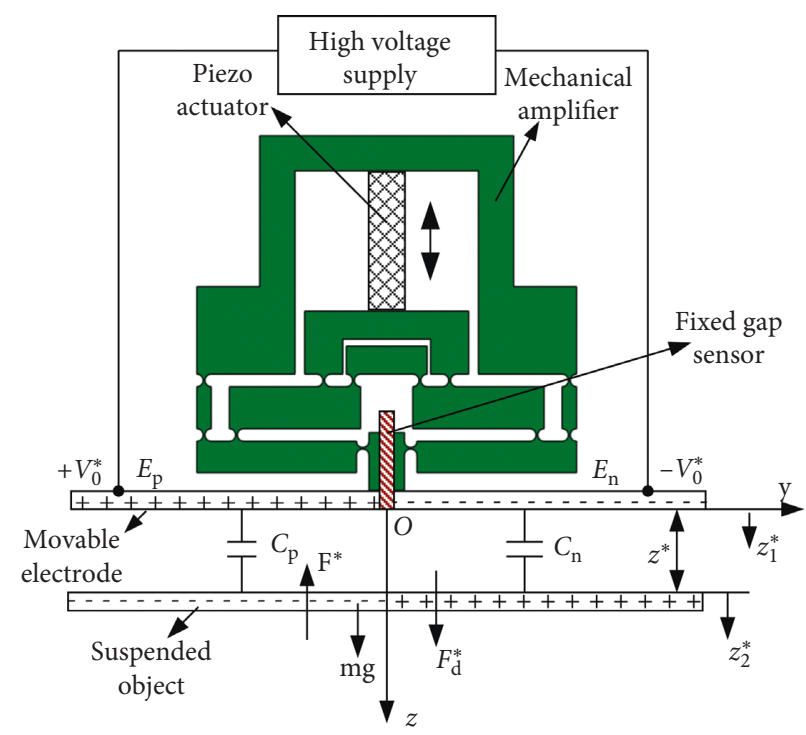

FIgURe 1: Model of electrostatic suspension system using PZT with one degree of freedom.

voltage to supply a control voltage to piezo actuator (PZT), and a mechanical amplifier. One end of the mechanical amplifier is fixed and another end is stuck with the movable electrode in the vertical plane. The displacement of the PZT actuator is as an input of the mechanical amplifier used to produce a large displacement of the movable electrodes. Two concentric movable electrodes, outer electrode $E_{\mathrm{p}}$ and inner electrode $E_{\mathrm{n}}$, are provided by constant positive voltage $\left(+V_{0}{ }^{*}\right)$ and constant negative voltage $\left(-V_{0}{ }^{*}\right)$, respectively. The areas of the two movable electrodes are same.

A fixed airgap sensor is placed at the geometrical center of the movable electrode and its sensitive head coincided with the origin $O$ of coordinate system $O x y z$ for the purpose to detect the position of the suspended object. The signal of this sensor is transmitted to the controller. Accordingly, the piezoelectric actuator is actuated by applying a drive voltage and generates a static displacement to the movable electrodes. The operation of the system is based on movement of electrodes caused by a piezo actuator, namely, as the actual position of suspended object respect to the fixed airgap sensor is greater than the reference gap, the suspension system requires the large enough electrostatic force to attract suspended object. This is performed by rapid movement of the movable electrode in direction where the gap length between the suspended object and the movable electrode decreased; as a consequence, the electrostatic force is increased. Similarly, as the actual position of suspended object is less than or equal to the reference gap, the movable electrode rapidly moves in the direction in which the gap between the movable electrode and the suspended object increased in order to make small electrostatic force; as a result of that, the electrostatic force is decreased.

\section{The Dynamic Motion of the Suspended Object}

The system modeling of the system will be derived using its simple electrical circuit representation as shown in Figure 1. 
In this modeling the electric resistances of components are ignored. The mass of the suspended object is defined as $m$. The symbols $z_{1}^{*}, z_{2}^{*}$ represent displacements of movable electrode and suspended object respect to the $\mathrm{Oz}$ axis, respectively. The difference between two displacements is denoted by $z^{*}$. The symbols $C_{\mathrm{p}}$ and $C_{\mathrm{n}}$ represent the variable capacitors formed by the suspended object and the positively-negatively charged electrode, respectively. The various forces acting on the suspended object in ambient air are the attractive electric, squeeze film damping, and gravitational forces. The external disturbance force is significantly small and can be ignored.

3.1. The Electrostatic Force. When the overlapping area of the movable electrodes and the suspended object is large enough compared with their differential gap length, the capacitance can be approximated as $[5,6]$

$$
C_{i}=\frac{\varepsilon A}{\left(z_{2}^{*}-z_{1}^{*}\right)}, \quad i=n, p .
$$

Let us assume the air leakage resistance in the airgap is neglected so that the only impedance element considered is capacitance. Based on the assumptions stated above, the electrostatic force $F^{*}$ can be expressed in terms of the gap length and the potential difference between the electrode and the object as follows:

$$
F^{*}=\frac{1}{2} \varepsilon A\left(\frac{V_{0}^{*}}{z_{2}^{*}-z_{1}^{*}}\right)^{2},
$$

where $V_{0}^{*}$ is the constant supplied voltage to the movable electrodes. We denote $\varepsilon=8.854 \times 10^{-12} \mathrm{~F} / \mathrm{m}$ and $A$ as the permittivity and the total area of electrodes, respectively. Equation (2) shows that the force $F^{*}$ is inversely proportional to the square of the gap length between the suspended object and the electrode $\left(z^{*}{ }_{2}-z^{*}{ }_{1}\right)$. This implies that the motion of the suspended object will exhibit an unstable behavior without any active control of the force $F^{*}$. Figure 2 shows the simulation of electrostatic force generated between movable electrode and the suspended object (4-inch silicon wafer) verse to the gap length. It shows that the electrostatic force can be controlled by changing the gap length between them.

3.2. The Damping Force. The ratio of the gap length between the suspended object and the electrode to the overlapping area of the object and the electrode is small; the air in the gap can be modeled as a squeeze film when the object moves vertically.

Assuming that the compressibility of air can be neglected and considering a one-dimensional case, damping force $F_{d}^{*}$ can be approximately expressed:

$$
F_{\mathrm{d}}^{*}=\int_{0}^{r_{0}} p(r, t) 2 \pi r \mathrm{~d} r=-\frac{3 \pi \eta r_{0}^{4}}{2} \frac{\left(\dot{z}_{2}^{*}-\dot{z}_{1}^{*}\right)}{\left(z_{2}^{*}-z_{1}^{*}\right)^{3}}
$$

where $p(r, t)$ is the pressure distribution in a thin film between electrode and suspended object, $\eta=18 \times 10^{-6} \mathrm{~N} \cdot \mathrm{s} / \mathrm{m}^{2}$ is the viscosity of air, and $r_{0}$ is a radius

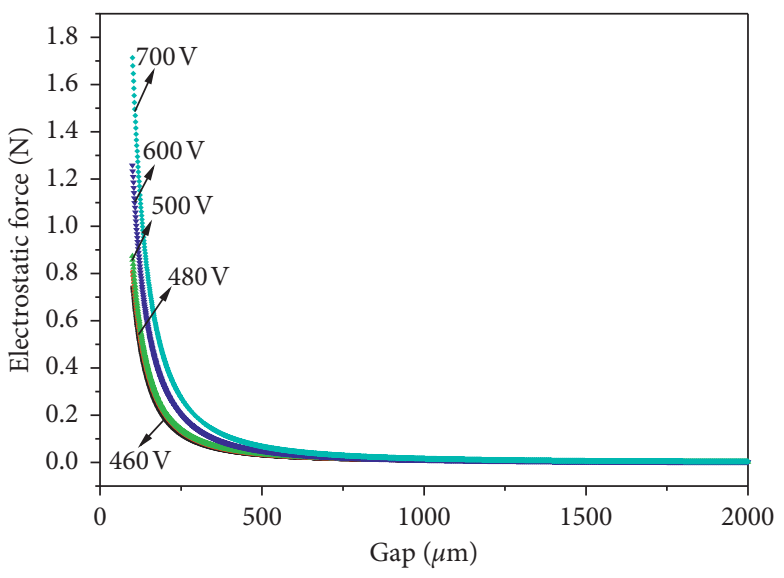

FIGURE 2: Simulation of electrostatic force verse to gap length.

of the suspended object. Due to rapid deformation of PZT, the movement time which the electrode needs to reach the equilibrium point is very short. This is implied that the suspended object is moving while the electrode has stopped. With this assumption, we can write as

$$
\dot{z}_{2}^{*}-\dot{z}_{1}^{*} \cong \dot{z}_{2}^{*} \text {. }
$$

Then, the damping force can be calculated as follows:

$$
F_{\mathrm{d}}^{*}=-\frac{3 \pi \eta r_{0}^{4}}{2} \frac{\dot{z}_{2}^{*}}{\left(z_{2}^{*}-z_{1}^{*}\right)^{3}} .
$$

Using $F^{*}$ and $F_{\mathrm{d}}^{*}$, the dynamic equation of the suspended object is described as

$$
m \ddot{z}_{2}^{*}=m g-\frac{1}{2} \varepsilon A\left(\frac{V_{0}^{*}}{z_{2}^{*}-z_{1}^{*}}\right)^{2}-\frac{3 \pi \eta r_{0}^{4}}{2} \frac{\dot{z}_{2}^{*}}{\left(z_{2}^{*}-z_{1}^{*}\right)^{3}} .
$$

3.3. The Dynamic Modeling of the Suspension System. The drawback of PZT is its small stroke or small strain to about $0.1 \%$ of its length. Therefore, a mechanical amplifier to produce a large displacement of the movable electrode is used. In our electrostatic suspension system, the principle amplification mechanics as shown in Figure 1 is employed. Figure 3 shows the prototype piezoelectric actuator-mechanical amplifier movable electrode set used in this study.

The PZT actuator is a kind of multilayer piezoelectric type to produce a large stroke using a small voltage. Compared to conventional piezoelectric actuators, they are smaller in size but can generate higher displacement and force at low voltage. The static displacement of PZT is obtained in the way that by applying a voltage $V_{\mathrm{cc}}^{*}$ to the PZT actuator. It can be expressed without considering hysteresis as

$$
\xi_{s}=d_{33} n V_{c c}^{*}
$$

where $\xi_{\mathrm{s}}$ is static displacement $(\mathrm{m}), d_{33}$ is the strain coefficient (field and displacement in polarization direction) $(\mathrm{m} /$ $\mathrm{V}), n$ is the number of ceramic layers, and $V_{c c}^{*}$ is operating voltage $(\mathrm{V})$. 


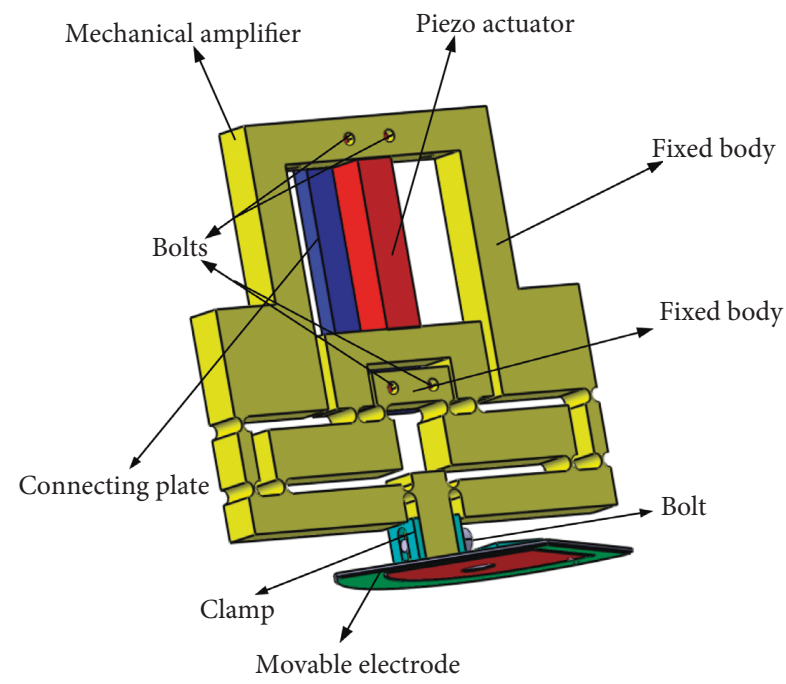

FIgURE 3: The piezoelectric actuator-mechanical amplifier movable electrode set.

The frequency response of the mechanical amplifierpiezo actuator-movable electrode unit was obtained by experiments using a dynamic analyzer (HP35665A, HP) as $288 \mathrm{~Hz}$. In other experiments, the frequency analysis of the control input signal obtained during a stable suspension was investigated, and it was shown that the frequency at which the amplitude of the control input signal is largest is about $60 \mathrm{~Hz}$, which is a much lower value than $288 \mathrm{~Hz}$. Therefore, the dynamic characteristic can be neglected, and the position of the movable electrode can be calculated as the product of the displacement of the piezoelectric actuator and the amplification factor of the mechanical amplifier. The static displacement of the movable electrode can be presented as follows:

$$
z_{1}^{*}=f d_{33} n V_{c c}^{*}
$$

where $f$ is the amplification factor of the mechanical amplifier.

In summary, the dynamic model for the system can be described as

$$
\begin{aligned}
m \ddot{z}_{2}^{*}= & \mathrm{mg}-\frac{1}{2} \varepsilon A\left(\frac{V_{0}^{*}}{z_{2}^{*}-f d_{33} n V_{\mathrm{cc}}^{*}}\right)^{2} \\
& -\frac{3 \pi \eta r_{0}^{4}}{2} \frac{\dot{z}_{2}^{*}}{\left(z_{2}^{*}-f d_{33} n V_{\mathrm{cc}}^{*}\right)^{3}} .
\end{aligned}
$$

3.4. Linearization. The purpose is to suspend the object at reference gap without any mechanical contact. In this system, this goal is performed based on active control electrostatic force around the equilibrium position by using the displacement of movable electrode as the input. In order easily to obtain the solution, the origin of the coordinate system is chosen to coincide with the fixed sensor head, and the initial position of the movable electrodes is in zero position as shown in Figure 1. The linearization technique will be applied to the nonlinear equation of electrostatic and damping force to obtain a linearization equation of motion of the suspended object at the equilibrium position.

Let's $z^{*}=z_{2}^{*}-z_{1}^{*}$, equation (2), and (5) can be written:

$$
\begin{aligned}
& F^{*}=\frac{1}{2} \varepsilon A\left(\frac{V_{0}^{*}}{z^{*}}\right)^{2}, \\
& F_{\mathrm{d}}^{*}=-\frac{3 \pi \eta r_{0}^{4}}{2} \frac{\dot{z}_{2}^{*}}{\left(z^{*}\right)^{3}} .
\end{aligned}
$$

Let's $z_{0}, z_{10}, z_{20}, V_{\mathrm{cc} 0}, F_{0}$, and $F_{\mathrm{d} 0}$ be the values at the equilibrium state, and $z, z_{1}, z_{2}, V_{\mathrm{cc}}, F$, and $F_{\mathrm{d}}$ be the linearization values of $z^{*}, z_{1}^{*}, z_{2}^{*}, V_{\mathrm{cc}}^{*}, F^{*}$, and $F_{\mathrm{d}}^{*}$. Then, the variables $z, z_{1}, z_{2}, \mathrm{~V}_{\mathrm{cc}}, F$, and $F_{\mathrm{d}}$ can be written:

$$
\begin{aligned}
z^{*} & =z+z_{0}, \\
z_{1}^{*} & =z_{1}+z_{10}, \\
z_{2}^{*} & =z_{2}+z_{20}, \\
V_{c c}^{*} & =V_{c c 0}+V_{c c}, \\
F^{*} & =F_{0}+F \\
F_{d}^{*} & =F_{d 0}+F_{\mathrm{d}}, \\
z_{0} & =z_{20}-z_{10} .
\end{aligned}
$$

In order to get a simple solution, the constant voltage supplied to movable electrode is chosen as $V_{0}^{*}=z_{20} \sqrt{2 \mathrm{mg} / \varepsilon A}$, where $z_{20}$ is the reference gap (i.e., the linearization value of displacement of suspended object respect to the origin $O$ ). Since the electrostatic force must be equal to the gravitational force at the equilibrium position, from equation (10) it obtained as $z_{10}=0$, and hence, $V_{\mathrm{cc} 0}=0$. Addition, the damping force is equal to zero at the equilibrium position, i.e., $F_{\mathrm{d} 0}=0$, so the linearization equations of electrostatic force and damping force, respectively, are obtained as

$$
\begin{aligned}
& F^{*}=F_{0}-k_{\mathrm{s}} z, \\
& F_{\mathrm{d}}^{*}=F_{\mathrm{d}}+F_{\mathrm{d} 0}
\end{aligned}
$$

where

$$
\begin{aligned}
& k_{\mathrm{s}}=\varepsilon A\left(\frac{V_{0}^{*^{2}}}{z_{20}^{3}}\right), \\
& k_{\mathrm{d}}=-\frac{3 \pi \eta r_{0}^{4}}{2 z_{20}^{3}}, \\
& F_{0}=\frac{1}{2} \varepsilon A\left(\frac{V_{0}^{*}}{z_{20}}\right)^{2} .
\end{aligned}
$$

We obtained the linearization equation of motion of suspended object as follows

$$
m \ddot{z}_{2}=\mathrm{mg}-F_{0}+k_{\mathrm{s}} z-F_{\mathrm{d}} .
$$

At the equilibrium point, $F_{0}=\mathrm{mg}$. So the linearization equations of motion of the system are obtained from equations (8) and (15) as 


$$
\left\{\begin{array}{l}
z_{1}=\operatorname{fnd}_{33} V_{\mathrm{cc}}, \\
m \ddot{z}_{2}=k_{s} z-k_{\mathrm{d}} \dot{z}_{2},
\end{array}\right.
$$

where $z=z_{2}-z_{1}$.

Equation (16) can be rewritten as follows:

$$
m \ddot{z}_{2}=k_{\mathrm{s}}\left(z_{2}-z_{1}\right)-k_{\mathrm{d}} \dot{z}_{2} .
$$

It is obtained as

$$
m \ddot{z}_{2}=k_{\mathrm{s}} z_{2}-k_{\mathrm{d}} \dot{z}_{2}-k_{\mathrm{s}} \mathrm{fnd}_{33} V_{\mathrm{cc}} .
$$

Since the damping force is proportional to the velocity of suspended object, it can be assumed that the damping force is very small at the equilibrium point and it can be ignored, i.e., $k_{\mathrm{d}} \dot{z}_{2} \approx 0$. Thus, equation of motion of suspended object can be written as follows:

$$
m \ddot{z}_{2}=k_{\mathrm{s}} z_{2}-k_{\mathrm{Vcc}} V_{\mathrm{cc}} .
$$

where $k_{\mathrm{Vcc}}=k_{\mathrm{s}} k_{\mathrm{pzt}}$ with $k_{\mathrm{pzt}}=$ fnd $_{33}$. The coefficient $k_{\mathrm{s}}$ is calculated by using equation (13) while the value of $k_{\mathrm{pzt}}$ is identified by using experimental data.

The plant in equation (19) with the transfer function is $1 /\left(k_{\mathrm{s}} / k_{\mathrm{Vcc}}-\left(m / k_{\mathrm{Vcc}}\right) s^{2}\right)$ is used to design a proximate time optimal controller for the electrostatic suspension system using the piezo actuator system. It is noted that plant is unstable plant with real characteristic roots $\pm \sqrt{k_{\mathrm{s}} / m}$. For unstable plants, a term called the recoverable set needs to be defined, and it is presented in the section below.

\section{Continuous Proximate Time Optimal Control for Suspension System}

4.1. Design of Continuous Proximate Time Optimal Control. In order to actively control electrostatic force of the electrostatic suspension system, the displacement of electrode caused by PZT actuator is used as input of the control scheme. Based on this control strategy, we proposed the control scheme for system based on states space as Figure 4 with $z_{21}$ and $z_{22}$ as the displacement and the velocity of the suspended object, respectively.

It is desirable to have a minimum time response to suspend silicon wafer at reference gap. However, ideal time optimal controllers that are bang-bang type are not good in practice, because they are sensitive to disturbance, parameter variations, and unmodeled dynamics [22, 23]. Because of this undesirable behavior, the continuous proximate time optimal control is applied in our electrostatic suspension system.

We define the state space as follows:

$$
\begin{aligned}
& z_{21}(t)=z_{2}^{*}(t)-z_{0}, \\
& z_{22}(t)=\dot{z}_{2}(t) .
\end{aligned}
$$

where $z_{21}(t)$ is the position error with $z_{0}$ being the desired final position of suspended object.

Thus, the state space of electrostatic suspension system in terms of new variables can be expressed as

$$
\left\{\begin{array}{l}
\dot{z}_{21}=z_{22}, \\
\dot{z}_{22}=\left(\frac{k_{\mathrm{s}}}{m}\right) z_{21}-\left(\frac{k_{\mathrm{Vcc}}}{m}\right) V_{\mathrm{cc}} .
\end{array}\right.
$$

Let $\omega_{n 0}^{2}=k_{\mathrm{s}} / m, k=k_{\mathrm{s}} / k_{\mathrm{Vcc}}, u(t)=V_{\mathrm{cc}},|u(t)| \leq V_{\mathrm{ccmax}}$ where $V_{\text {ccmax }}$ is the maximum control effort. The state space in equation (21) can be written as

$$
\dot{z}=A z+B u(t)
$$

where

$$
\begin{aligned}
& z=\left[\begin{array}{ll}
z_{21} & z_{22}
\end{array}\right]^{T}, \\
& A=\left[\begin{array}{cc}
0 & 1 \\
\omega_{n 0}^{2} & 0
\end{array}\right],
\end{aligned}
$$

$$
B=\left[\begin{array}{c}
0 \\
-\left(\frac{\omega_{n 0}^{2}}{k}\right)
\end{array}\right] .
$$

The recoverable set and the discontinuous time optimal control law for the system, respectively, are obtained as $[24,25]$

$$
\begin{aligned}
\mathrm{RS}_{z} & =\left\{\left|\frac{z_{21}+z_{22}}{\sqrt{k_{\mathrm{s}} / m}}\right|<V_{\mathrm{ccmax}}\left(\frac{k_{\mathrm{V} c \mathrm{c}}}{k_{\mathrm{s}}}\right)\right\}, \\
u(z) & = \begin{cases}u_{\mathrm{max}} \operatorname{sign}\left(z_{21}-Z_{21}\left(z_{22}\right)\right), & \text { if }\left(z_{21}-Z_{21}\left(z_{22}\right)\right) \neq 0, \\
u_{\max } \operatorname{sign}\left(z_{22}\right), & \text { if }\left(z_{21}-Z_{21}\left(z_{22}\right)\right)=0,\end{cases}
\end{aligned}
$$




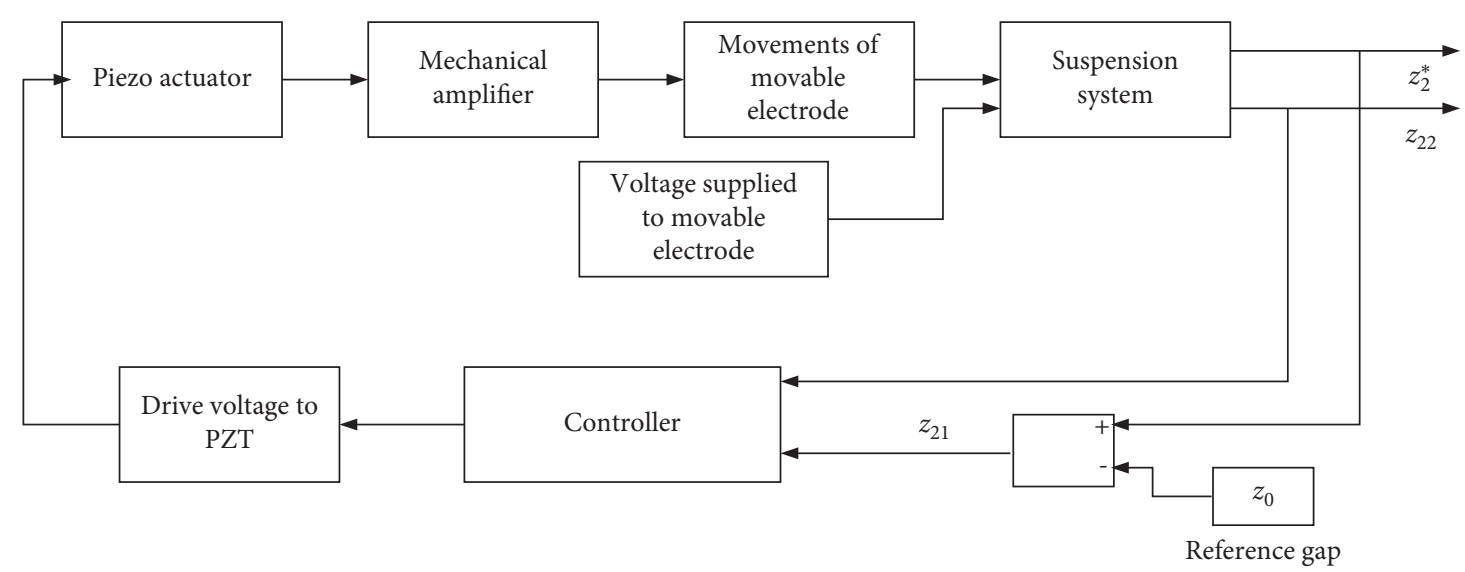

FIGURE 4: Scheme control of electrostatic suspension system.

where

$$
\begin{aligned}
Z_{21}\left(z_{22}\right) & =\left(\frac{k_{\mathrm{Vcc}}}{k_{\mathrm{s}}}\right) \operatorname{sign}\left(z_{22}\right)\left(1-\sqrt{1+\left(\frac{m k_{\mathrm{s}}}{k_{\mathrm{Vcc}}^{2}}\right) z_{22}^{2}}\right), \\
u_{\max } & =V_{\mathrm{ccmax}} .
\end{aligned}
$$

Based on the above discontinuous time optimal control law, the continuous proximate time optimal control for electrostatic suspension using piezo actuator is proposed as follows [22]:

$$
U(z)=\operatorname{sat}\left\{p_{1}\left(z_{21}-Z_{21}\left(z_{22}\right)\right)+\operatorname{sat}\left(p_{1} p_{2} z_{22}\right)\right\},
$$

where $p_{1}$ and $p_{2}$ are arbitrary positive gain constants and the function $\operatorname{sat}(\sigma)$ is defined as

$$
\operatorname{sat}(\sigma)= \begin{cases}u_{\max } \operatorname{sign}(\sigma), & \text { for }|\sigma| \geq u_{\max }, \\ \sigma, & \text { for }|\sigma|<u_{\max }\end{cases}
$$

Claim 1. For all $z \neq 0, U(z) \longrightarrow u(z)$ as $p_{1} \longrightarrow \infty$. This claim is proven as follows:

(1) In case of $z_{21}-Z_{21}\left(z_{22}\right)=0$, the control law in equation (27) becomes $U(z)=\operatorname{sat}\left\{\operatorname{sat}\left(p_{1} p_{2} z_{22}\right)\right\}$. It is easily obtained as $p_{1} \longrightarrow \infty, U(z)=$ $u_{\max } \operatorname{sign}\left(z_{22}\right)$.

(2) In case of $z_{21}-Z_{21}\left(z_{22}\right) \neq 0$, as $p_{1} \longrightarrow \infty$ then $\left|p_{1}\left(z_{21}-Z_{21}\left(z_{22}\right)\right)\right| \gg>\operatorname{sat}\left(p_{1} p_{2} z_{22}\right)$, which it gives as $U(z)=u_{\max } \operatorname{sign}\left(z_{21}-Z_{21}\left(z_{22}\right)\right)$.

In other words, the ideal time optimal control in equation (27) is a special case of the continuous proximate time optimal control defined in equation (27) if $p_{1}$ is large enough.

Claim 2. If $\|z\|$ is small, then $U(z)=u(z)=\widehat{p}_{1}\left(z_{21}+\widehat{p}_{2} z_{22}\right)$, where $\widehat{p}_{1}, \widehat{p}_{2}$ are arbitrary gains constants. This claim is proven as follows:

(1) In case $\|z\|$ is small, that means $z_{21}, z_{22}$ are small, and sat $(z)=z$. Thus, the control law in (27) becomes

$$
U(z)=p_{1}\left(z_{21}-Z_{21}\left(z_{22}\right)\right)+p_{1} p_{2} z_{22}
$$

Since $z_{22}$ is very small, we can assume that

$$
\sqrt{1+\left(\frac{m k_{\mathrm{s}}}{k_{\mathrm{V} c \mathrm{c}}^{2}}\right) z_{22}^{2}}=1
$$

It means that $Z_{21}\left(z_{22}\right)=0$. Therefore, equation (29) becomes

$$
U(z)=p_{1}\left(z_{21}+p_{2} z_{22}\right)
$$

Let $\widehat{p}_{1}=p_{1}, \widehat{p}_{2}=p_{2}$, we can write equation (31) in terms of $\widehat{p}_{1}$ and $\widehat{p}_{2}$ as follows:

$$
U(z)=\widehat{p}_{1}\left(z_{21}+\widehat{p}_{2} z_{22}\right) .
$$

The claim 2 is proven. It implied that the linear control in equation (32) is a special case of the continuous proximate time optimal control defined in equtaion (27) if the state space is small enough.

4.2. Stability Analysis of the Control for System. In this section, we establish restriction on the design of constants $\widehat{p}_{1}$ and $\widehat{p}_{2}$ to guarantee global stability of the system presented in equation (21) with the feedback control in equation (27). In order to easily get a solution and to have more simple equations, without loss generally, we assume $u_{\max }=1$. We introduce the following notation:

$$
\begin{aligned}
U & =\left\{z \in R^{2},|\mu(z)|<1\right\}, \\
U^{-} & =\left\{z \in R^{2}, \mu(z) \leq-1\right\}, \\
U^{+} & =\left\{z \in R^{2}, \mu(z) \geq 1\right\},
\end{aligned}
$$

where

$$
\mu(z)=\left\{p_{1}\left(z_{21}-Z_{21}\left(z_{22}\right)\right)+\operatorname{sat}\left(p_{1} p_{2} z_{22}\right)\right\} .
$$

The regions above terms in the phase plant are shown in Figure 5. The following stability analysis consists of three parts, which are motivated in some respect by Kaylon [22], Pao and Franklin [26], and Workman et al. [27]. 


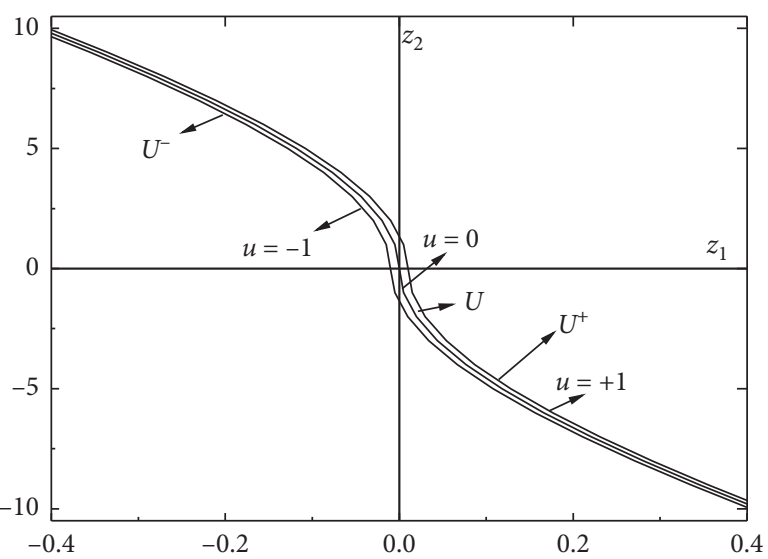

FIgURE 5: Regions $U, U^{+}$, and $U^{-}$in the phase plan.

Part 1. All trajectories entered into the region $U$ in some finite time.

In the previous section, it is shown that the ideal bangbang control for electrostatic suspension having plant presented in (21) exists if initial states of system are in the recoverable set. In addition, the switching curve is inside the region $U$ as shown in Figure 6. Therefore, all trajectories have to enter into the region $U$ in some finite time.

Part 2. When the state system is in domain $U$, it remains in domain $U$.

The region $U$ is bounded from above by the boundary on which $u=+1$ and bounded from below by the boundary on which $u=-1$ as shown in Figure 5. We know that when $z \in U$, by using a saturation function is defined in equation (27), we can conclude that $U(z)=\mu(z)$. Thus, if the sign of $\dot{\mu}(z)$ is opposite that of control signal $u$, then the system state will move to the interior of $U$. Therefore, in order to prove

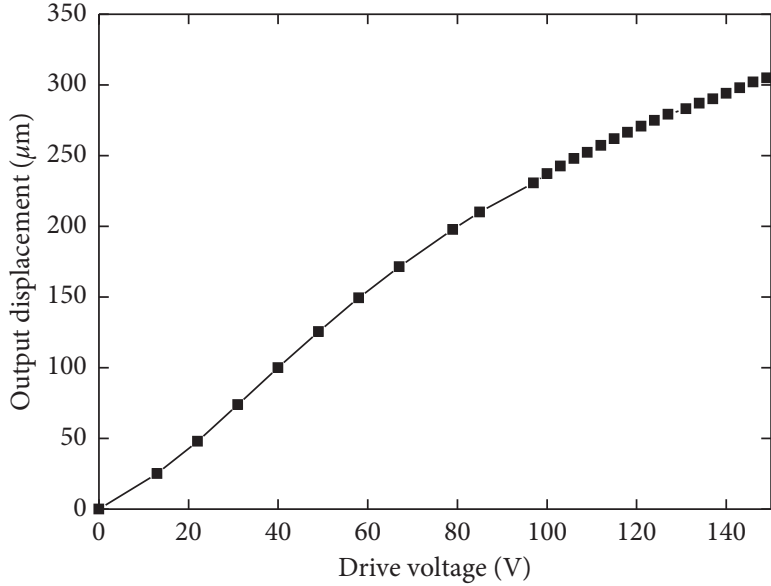

FIGURE 6: Output displacement of mechanical amplifier verse to drive voltage supplied to piezo actuator.

that any trajectory originating within $U$ remains the interior of $U$, we must show that $\dot{\mu}(z) \leq 0$ where $\mu(z)=+1$ and $\dot{\mu}(z) \geq 0$ where $\mu(z)=-1$

Part 3. This part shows that there exists a Lyapunov function $V(z)$ when the state system is in the $U$-region.

The candidate Lyapunov function is chosen as follows:

$$
V(z)=C_{1} z_{21}^{2}+C_{2} z_{22}^{2}, \quad C_{1}>0, C_{2}>0 .
$$

We found that

$$
\dot{V}(z)=2 C_{1} z_{21} \dot{z}_{21}+2 C_{2} z_{22} \dot{z}_{22}
$$

Substituting the state equation in equation (21) into equation (36), and noting that $U(z)=\mu(z)$, we obtained

$$
\dot{V}(z)=2 z_{21} z_{22}\left(C_{1}-C_{2} \omega_{n 0}^{2}\left(\frac{p_{1}}{k-1}\right)\right)+2 C_{2}\left(\left(\frac{\omega_{n 0}^{2} z_{22} \operatorname{sign}\left(z_{22}\right)}{k^{2}}\right)\left(1-\sqrt{1+\left(\frac{k}{\omega_{n 0}^{2}}\right)^{2} z_{22}^{2}}\right)-\left(\frac{\omega_{n 0}^{2}}{k}\right) z_{22} \operatorname{sat}\left(p_{1} p_{2} z_{22}\right)\right) .
$$

We choose $C_{1}=C_{2} \omega_{n 0}^{2}\left(p_{1} / k-1\right)$ and $p_{1}>k$. The above equation becomes

$$
\dot{V}(z)=2 C_{2}\left(\left(\frac{\omega_{n 0}^{2} z_{22} \operatorname{sign}\left(z_{22}\right)}{k^{2}}\right)\left(1-\sqrt{1+\left(\frac{k}{\omega_{n 0}^{2}}\right)^{2} z_{22}^{2}}\right)-\left(\frac{\omega_{n 0}^{2}}{k}\right) z_{22} \operatorname{sat}\left(p_{1} p_{2} z_{22}\right)\right) .
$$

Since $z_{22} \operatorname{sign}\left(z_{22}\right)>0$ and $z_{22} \operatorname{sat}\left(k_{1} k_{2} z_{22}\right)>0$, then

$$
\dot{V}(z)<0 \text {. }
$$

It is based on equations (39) and (40), we can conclude that the system is stable when the state system is in the $U$ region. 
The combination of three part of above section proves that the system is stable.

In other hand, the claim II shown that when $\|z\|$ is small, the control law in equation (25) converges to the linear control in equation (30). In this case, we can rewrite as

$$
U(z)=p z=\left[\begin{array}{ll}
p_{1 L} & p_{2 L}
\end{array}\right]\left[\begin{array}{c}
z_{21} \\
z_{22}
\end{array}\right]
$$

where $p=\left[\begin{array}{ll}p_{1 L} & p_{2 L}\end{array}\right], p_{1 L}=\widehat{p}_{1}$, and $p_{2 L}=\widehat{p}_{1} \widehat{p}_{2}$ are the gains of the linear control.

Replacing above equation into state space of system in equation (21), we obtained as

$$
\dot{z}=(A-B p) z \text {. }
$$

The characteristic equation becomes

$$
\widehat{A}=|s I-A+B p|=s^{2}+\frac{\omega_{n 0}^{2} \widehat{p}_{1} \widehat{p}_{2}}{k} s-\left[\omega_{n 0}^{2}-\left(\frac{\omega_{n 0}^{2}}{k}\right) \widehat{p}_{1}\right]=0 .
$$

Routh's array in that case is

$$
\left[\begin{array}{cc}
1 & -\left[\omega_{n 0}^{2}-\left(\frac{\omega_{n 0}^{2}}{k}\right) \widehat{p}_{1}\right] \\
\frac{\omega_{n 0}^{2} \widehat{p}_{1} \widehat{p}_{2}}{k} & 0 \\
-\left[\omega_{n 0}^{2}-\left(\frac{\omega_{n 0}^{2}}{k}\right) \widehat{p}_{1}\right]
\end{array}\right]
$$

The condition for ensuring the dynamic system in equation (41) is stable that all eigenvalues of $\widehat{A}$ to have negative real parts [20]. It implies that all coefficients in the first column of the Routh array have the same sign. In other words $\widehat{p}_{1}>k, \widehat{p}_{2}>0$. It means that $p_{1}>k$ and $p_{2}>0$.

4.3. Design of Linear Controller. Since the continuous proximate time optimal control will converge to the linear control when state space of the system near to the origin. Therefore, the design of linear control is necessary. The poles placement method is chosen. The poles are chosen depended on required overshoot and settling time of the system. The characteristic equation for the dominant poles is

$$
s^{2}+2 \xi \omega s+\omega^{2}=0
$$

where $\omega, \xi$ are the natural frequency and coefficient damping of closed system, respectively.

Equation (42) is compared with equation (44) we obtained as

$$
\begin{array}{r}
\frac{\omega_{n 0}^{2} \widehat{p}_{1} \widehat{p}_{2}}{k}=2 \xi \omega, \\
{\left[\omega_{n 0}^{2}-\left(\frac{\omega_{n 0}^{2}}{k}\right) \widehat{p}_{1}\right]=-\omega^{2} .}
\end{array}
$$

The above equations lead to that

$$
\begin{aligned}
& \widehat{p}_{1}=k\left(\frac{\omega^{2}}{\omega_{n 0}^{2}}+1\right), \\
& \widehat{p}_{2}=\frac{2 \xi \omega}{\left(\omega^{2}+\omega_{n 0}^{2}\right)} .
\end{aligned}
$$

Based on the above equation, the gains of linear control are calculated.

\section{Simulation and Experimental Works}

5.1. Design of Mechanical Amplifier and Movable Electrode. The mechanical amplifier is used to produce large displacement of movable electrodes since commercial piezo actuators have a limit deformation range of about $1 \mu \mathrm{m} / \mathrm{mm}$ [28]. A flexure hinge has often been used to amplify the displacement of a piezo actuator since it has the strengths of no backlash, not requiring lubrication [29]. Figure 1 presents a schematic of magnification mechanism. It is composed of four links in order to obtain two-step magnification [30]. In this study, the mechanical amplifier employs a right circular single-axis flexure hinge since it has some advantages [31].

The mechanical amplifier-piezo actuator-movable electrode unit consists of piezo actuator, lever type mechanical amplifier, and movable electrode as shown in Figure 3. Two fixed bodies of mechanical amplifier are connected by using a connecting plate. Movable electrode is connected to mechanical amplifier by using clamps and bolts. A multilayer piezoelectric actuator produced by NEC/TOKIN Co. (AE1010D44H40) is used. It has a maximum displacement of $(40 \pm 2) \mu \mathrm{m}$ at the maximum driving voltage of $150 \mathrm{~V}$, a natural frequency of $34 \mathrm{kHz}$, and a size of $10 \times 10 \times 40 \mathrm{~mm}^{3}$ [28].

Figure 6 presents the performance characteristic of the mechanical amplifier-piezo actuator-movable electrode unit in which the displacement of movable electrode is enlarged by using the mechanical amplifier. In this test, the voltage is monotonically increased from 0 to $150 \mathrm{~V}$ to measure the input of the piezo actuator and the output displacement of movable electrode. The output displacement of about $302 \mu \mathrm{m}$ is obtained at the maximum voltage of $150 \mathrm{~V}$ supplied to piezo actuator.

The value of the constant $k_{\text {pzt }}$ in equation (19) was identified by experimental results as $2.02 \times 10^{-6}$.

5.2. Determining of Voltage Values Supplied to Electrodes. Since the piezo actuator used in this study operates with only positive voltages, the value of constant voltage supplied to the movable electrode is an important value for operating of the suspension system. The reason is that the equilibrium position of the movable electrode, meaning the drive voltage supplied to piezo actuator, is depended on the constant voltage supplied to movable electrode. When the suspended object reaches the equilibrium point, the electrostatic force calculated by equation (1) must be equal to the gravitational force. It means that 


$$
\frac{1}{2} \varepsilon A\left(\frac{V_{0}^{*}}{z_{10}-z_{20}}\right)^{2}=\mathrm{mg} .
$$

Figure 7 (a) shows the initial position of the electrostatic suspension system using piezo actuator. The gaps $z_{20}$ and $z_{\text {in }}$ denote the desired suspension gap and the initial gap, respectively. In the initial position of suspension system, the movable electrode is in zero position respect to the $\mathrm{Oz}$ axis as shown in Figure 7(a). The different final equilibrium positions of movable electrode depended on constant voltage supplied to it can be presented as follows.

(a) If the voltage $V_{0}$ determined as $V_{0}=z_{20} \sqrt{2 \mathrm{mg} / \varepsilon A}$ is supplied to the movable electrode, i.e., $V_{0}^{*}=V_{0}$, then the movable electrode must be returned to its initial position at the equilibrium state as shown in Figure $7(\mathrm{~d})$. It means that the voltage applied to piezo actuator $V_{\mathrm{cc}}$ is equal to zero volts at this time.

(b) If the voltage supplied to the movable electrode is smaller than $V_{0}$, i.e., $V_{0}^{*}<V_{0}$, then the distance between the movable electrode and the suspended object at the equilibrium state must be smaller than $z_{20}$ as shown in Figure 7 (b). It means that the voltage supplied to the piezo actuator $V_{\mathrm{cc}}$ at the equilibrium point should be a positive value. The extended displacement of the piezo actuator in the equilibrium position in that case can be calculated as

$$
d_{\mathrm{PZT}}=\left(\frac{V_{0}-V_{0}^{*}}{f \sqrt{2 \mathrm{mg} / \varepsilon A}}\right) .
$$

(c) If the voltage supplied to movable electrode is larger than $V_{0}$, i.e. $V_{0}^{*}>V_{0}$, then distance between the movable electrode and suspended object at the equilibrium state must be bigger than $z_{20}$ as shown in Figure $7(\mathrm{c})$. It means that the voltage supplied to electrode $V_{\mathrm{cc}}$ at the equilibrium point should be negative value. The retracted displacement of piezo actuator in the equilibrium position in that case can be calculated as

$$
d_{\mathrm{PZT}}=-\left(\frac{V_{0}^{*}-V_{0}}{f \sqrt{2 \mathrm{mg} / \varepsilon A}}\right)
$$

The analysis results presented in Figure 8 show that the voltage supplied to the movable electrode should be less than the bias voltage $\left(V_{0}^{*}<480 \mathrm{~V}\right)$ to ensure the piezo actuator extends with positive displacement at its equilibrium position. However, this supplied voltage should not be too small as it will cause the extension of piezo actuator be over the working range.

5.3. The Prototype of Device. In order to implement contactless support by stabilizing the motion of a suspended object fully during the suspension process, several movable electrodes have to be used [25]. In this system, we use three independent movable electrode systems, i.e., mechanical amplifier-piezo actuator-movable electrode unit. The electrode set is arranged in a fan shape with an angle of $120^{\circ}$. The three outer electrodes together form a circle that has the same diameter as the suspended object. Figure 8 presents the fabricated prototype of the electrostatic static suspension system using piezo actuator. Each mechanical amplifierpiezo actuator-movable electrode unit is fixed on the upper plate by using bolts. The material used for the mechanical amplifier is duralumin. The copper electrodes are fabricated by using a standard wet chemical etching process. Fiber optical displacement sensors (PM-E, Nanotex Co.) are used as airgap sensors to measure the displacements of the suspended object.

5.4. Simulation Results. A 4-inch silicon wafer with radius, thickness, and mass of $50 \mathrm{~mm}, 0.71 \mathrm{~mm}$, and $9.1 \mathrm{~g}$, respectively, is used as the suspended object. The initial position of the suspended object is $330 \mu \mathrm{m}$ and the reference gap is $300 \mu \mathrm{m}$. In these simulations, the coefficient $k_{\text {pzt }}$ is identified by experiments as $2.02 \times 10^{-6} \mathrm{~V} / \mathrm{m}$ while the coefficient $k_{\mathrm{s}}$ is calculated by using equation (15) as 544.7. In order to prevent the saturation of the piezo actuator, the voltage control effort should be in the operating range of the piezo actuator, and they are set as $V_{\mathrm{ccmax}}=35 \mathrm{~V}$ and $V_{\mathrm{ccmin}}=0 \mathrm{~V}$. These driven voltages cause maximum and minimum predicted displacements of the movable electrode as $70 \mu \mathrm{m}$ and $0 \mu \mathrm{m}$, respectively. In order to ensure that the piezo actuator extends with positive displacements at its equilibrium position, the supplied voltage to movable electrode should be less than the bias voltage so it is chosen as $460 \mathrm{~V}$. The simulation parameters of the model are presented in Table 1.

The following simulations demonstrate the performance of the continuous proximate time optimal control. In those simulations, the gains $p_{1}, p_{2}$ of the linear controller are obtained as $4.2547 E 7$ and 104.5837, respectively. Figure 9 shows simulation results of movements of the electrode set (Figure 9(a)) and the suspended object (Figure 9(b)) during a suspension process. As shown in Figure 9(a), the control effort $V_{\text {ccmax }}$ is applied to piezo actuator immediately after the control starts causes the electrode set moves down rapidly to quickly increase the attractive force. After that, the electrode set moves up as the suspended object converges on the reference position.

As can be seen in Figure 10, where the switching part of the control response is zoomed, after the control effort approaches $V_{\text {ccmax }}$, it remains near $V_{\text {ccmax }}$ until the trajectory enters the final region. Figure 10 also reveals that once the trajectory enters the final region, it converges to the equilibrium position smoothly and remains there; the settling time for the initial condition tracks to the reference position is approximately of $38 \mathrm{~ms}$. The simulation results also show that the equilibrium position of movable electrode is $14 \mu \mathrm{m}$ where it is expected as in the Figure 7(b).

From the simulation results, it could be concluded that the designed proximate time optimal controller is really suited for the electrostatic suspension system using piezo actuators. By using that controller, the displacements of 


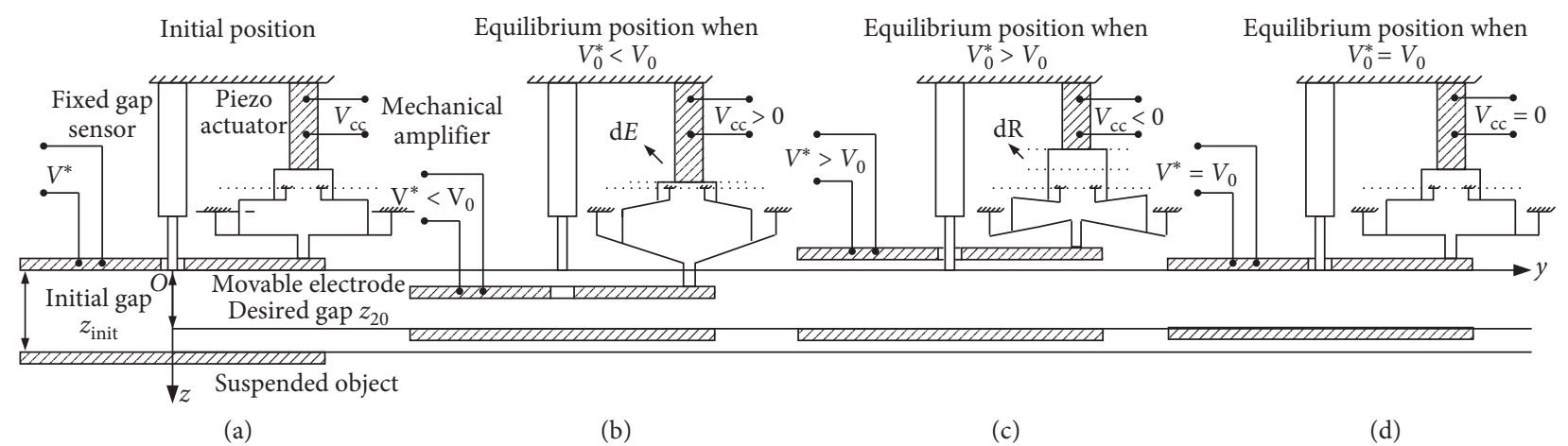

dE: extended displacement of PZT

dR: retracted displacement of PZT

FIgURE 7: Equilibrium positions of electrode depend on voltage supplied to it.

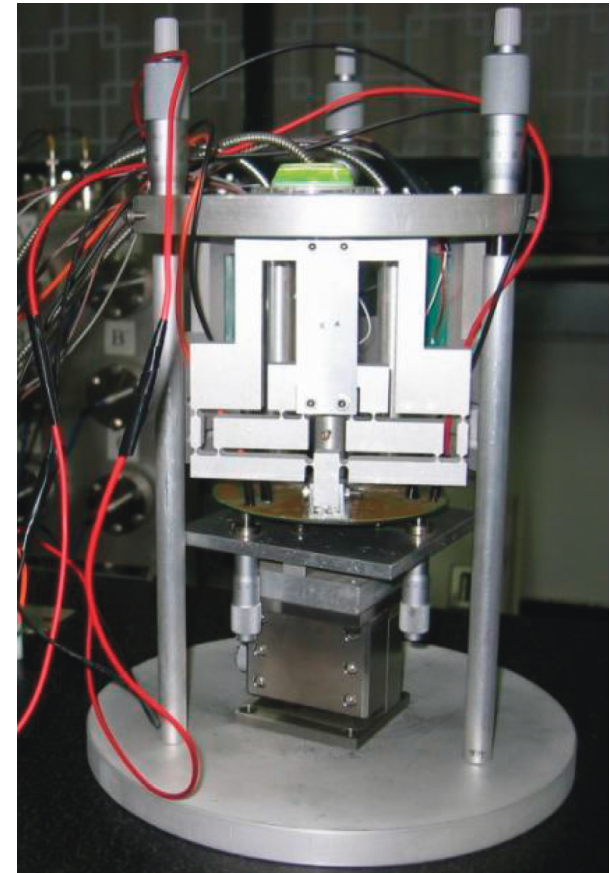

FIGURE 8: Electrostatic suspension system using piezo actuator.

TABLE 1: Simulation parameters of the model.

\begin{tabular}{lc}
\hline Parameters & Value \\
\hline Mass of 4-inch silicon wafer, $m$ & $9.1 \mathrm{~g}$ \\
Radius of 4-inch silicon wafer, $r_{0}$ & $50 \mathrm{~mm}$ \\
Total area of electrodes, $A$ & $7.85 \times 10^{3} \mathrm{~mm}^{2}$ \\
Initial gap length, $z_{\text {in }}$ & $330 \mu \mathrm{m}$ \\
Reference gap length, $z_{0}$ & $300 \mu \mathrm{m}$ \\
Constance voltage supplied, $V_{0}$ & $460 \mathrm{~V}$ \\
$k_{\text {pzt }}$ & $2.02 \times 10^{-6}(\mathrm{~V} / \mathrm{m})$ \\
$k_{s}$ & 544.7 \\
$V_{\text {ccmax }}$ & $35 \mathrm{~V}$ \\
$V_{\text {ccmin }}$ & $0 \mathrm{~V}$ \\
\hline
\end{tabular}

piezo actuators could be bounded in working ranges without saturation to control the suspended object levitated at the reference position.
5.5. Experimental Results Obtained by Using Proximate Time Optimal Control. This section presents experimental results of suspension by using continuous proximate time optimal controller to show the effectiveness of the proposed method. The controller was implemented digitally using a digital signal processor (DSP) system. The control sampling time was $50 \mu \mathrm{s}$. The voltage supplied to movable electrode is selected as the same in simulation $460 \mathrm{~V}$. In the experiments, the controller parameters are recalculated in which the parameter $m$ of mass should be one third of the mass of the silicon wafer, since three-electrode set exerts suspension forces on a single silicon wafer. It is noted that the area of each movable electrode set was $2.5 \times 10^{3} \mathrm{~mm}^{2}$.

Figure 11 shows the experimental results including movements of the silicon wafer measured by three position sensors and three movable electrodes \#1, \#2, and \#3 after the controller was switched on, respectively. Figures 11(a), 11(c), and 11(e)) reveal that the 4 -inch silicon wafer was suspended from the initial gap of $330 \mu \mathrm{m}$ to a stable state of suspension at a reference gap of $300 \mu \mathrm{m}$ with average amplitudes of fluctuation around $0.30 \mu \mathrm{m}$. A certain period of time of approximately $0.03 \mathrm{~s}$ was needed for the silicon wafer to start being lifted upwards from the initial gap length $330 \mu \mathrm{m}$. Figure 11 shows that there are little differences about equilibrium positions of the suspended object at 3 sensors \#1, \#2, \#3, namely, equilibrium positions at sensor \#1 is $300 \mu \mathrm{m}$, at sensor \#2 is $314 \mu \mathrm{m}$, and at sensor \#3 is $312 \mu \mathrm{m}$. This is obvious because the suspension is performed by using three independent electrode sets. The heterogeneity of the geometric dimensions of mechanical amplifiers due to manufacturing errors can cause this discrepancy.

Figures 11(b), 11(d), and 11(f)) show the variations of displacement of movable electrodes. Same as simulation results, movable electrodes move down rapidly immediately after the control starts from zero to maximum displacement approximately of $80 \mu \mathrm{m}$ to increase the attractive forces. After that, electrodes move up as the silicon wafer converges on the reference position. Once the position of the suspended object enters the final region, it converges to the equilibrium position smoothly and remains there. It shows that when the silicon wafer reached the stable suspension 


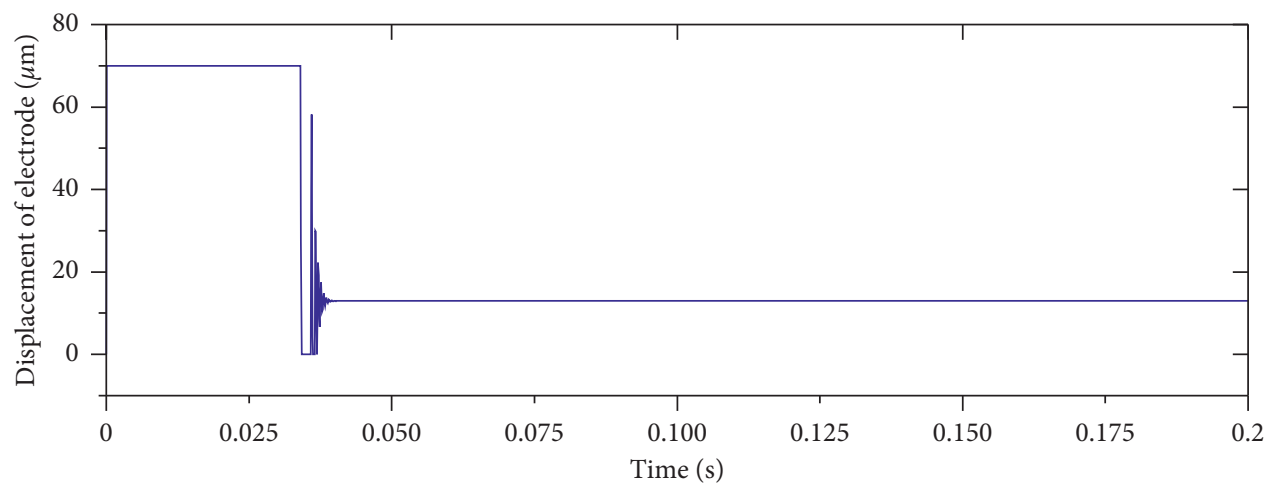

(a)

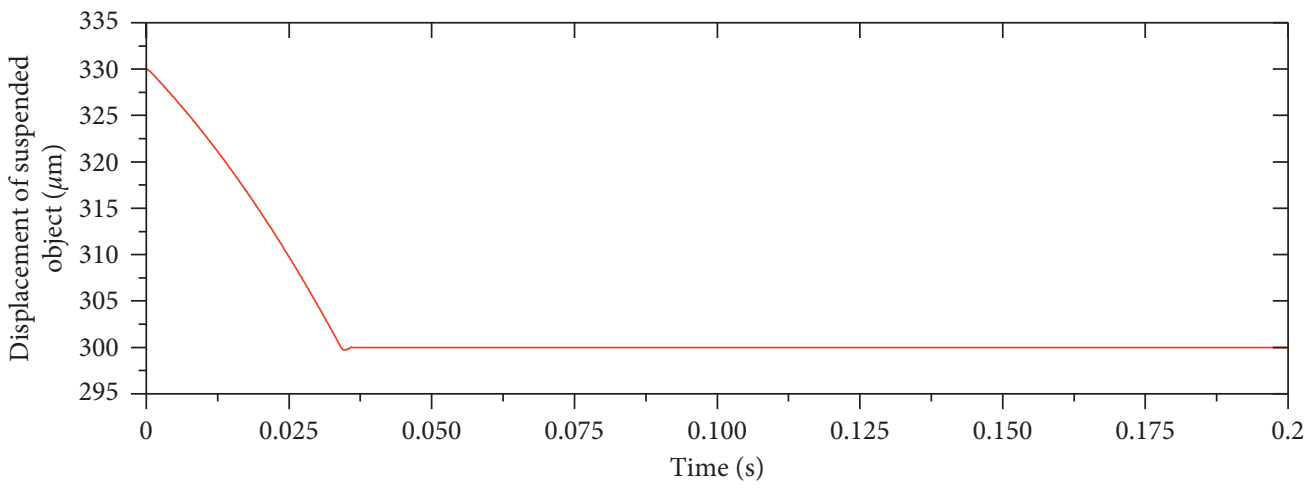

(b)

Figure 9: The response of the system with voltage supplied to electrode of $460 \mathrm{~V}$.

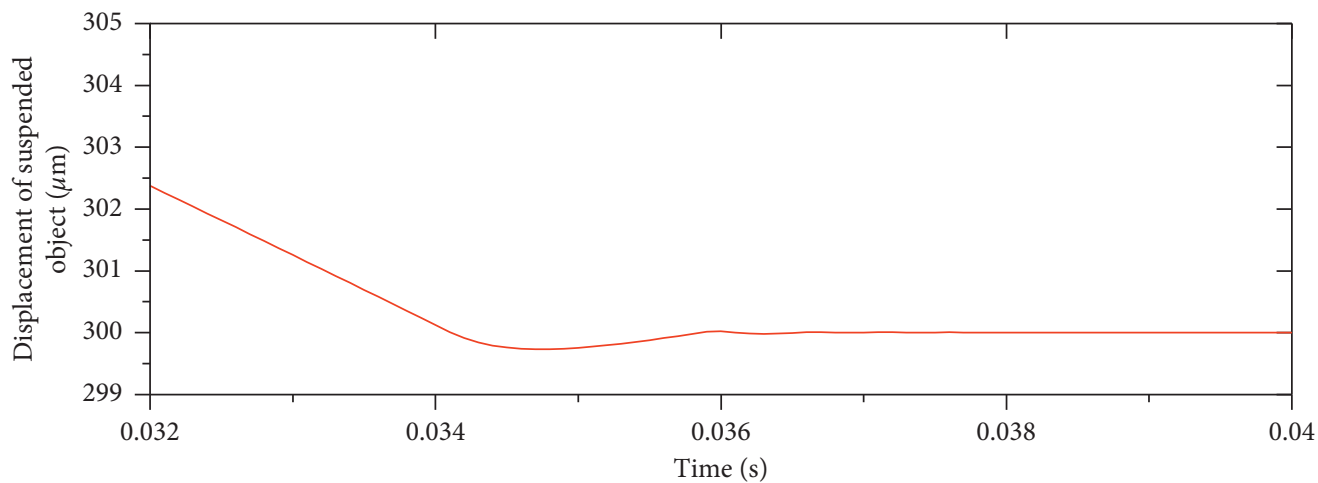

(a)

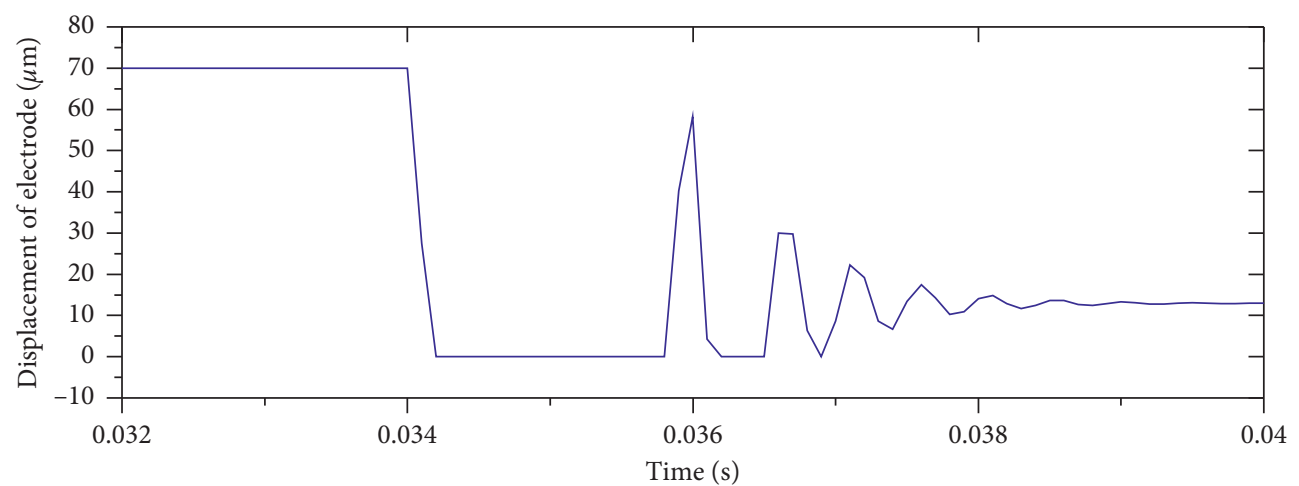

(b)

FIGURE 10: Zoomed version of the switching parts of the control response. 


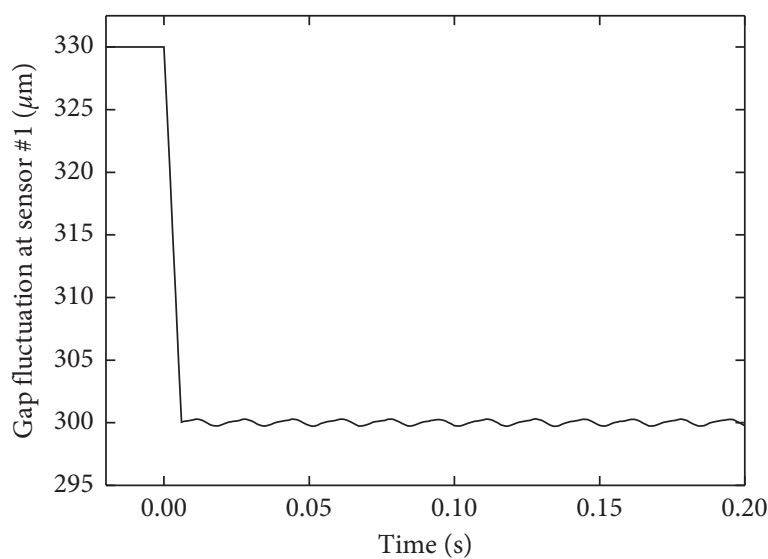

(a)

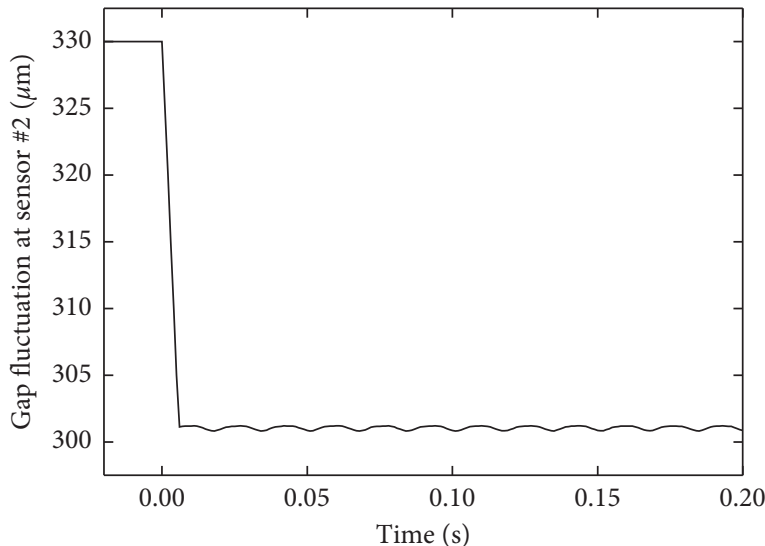

(c)

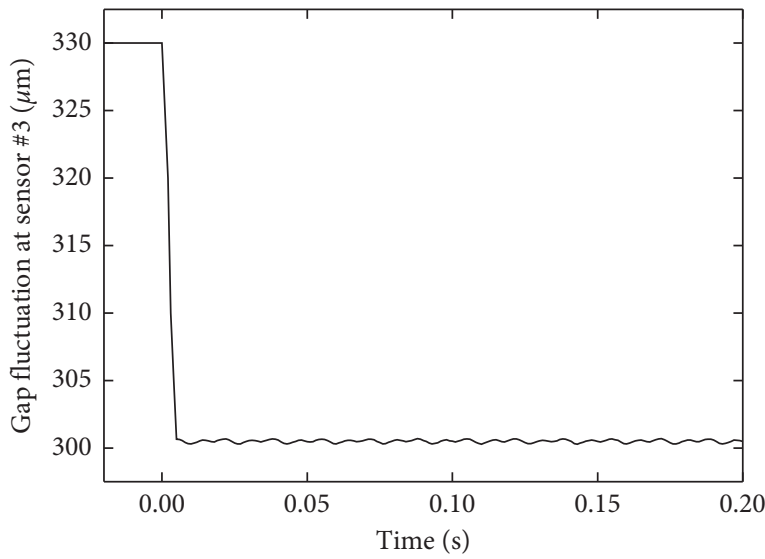

(e)

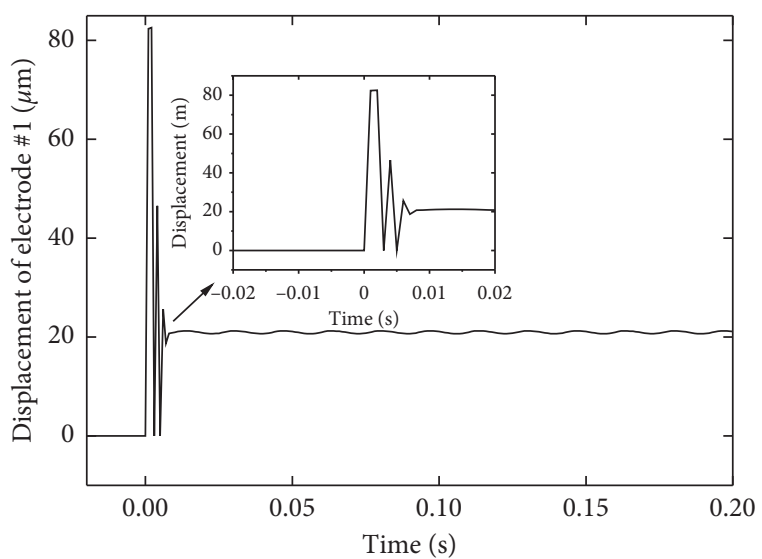

(b)

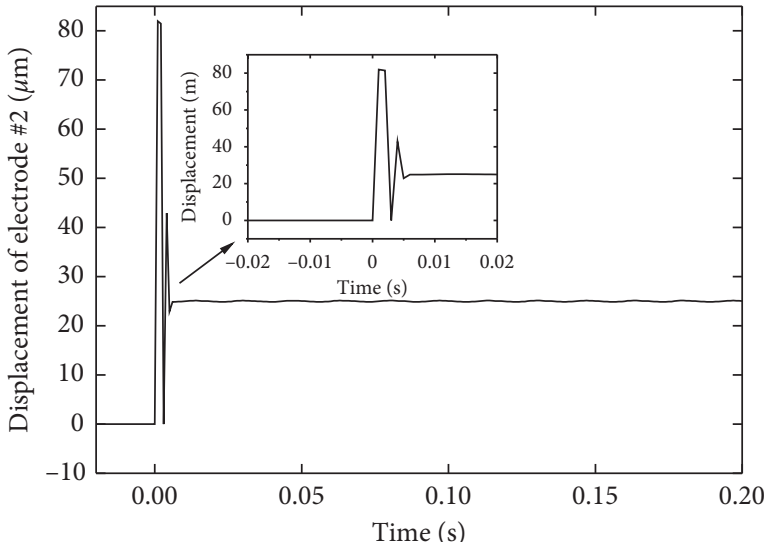

(d)

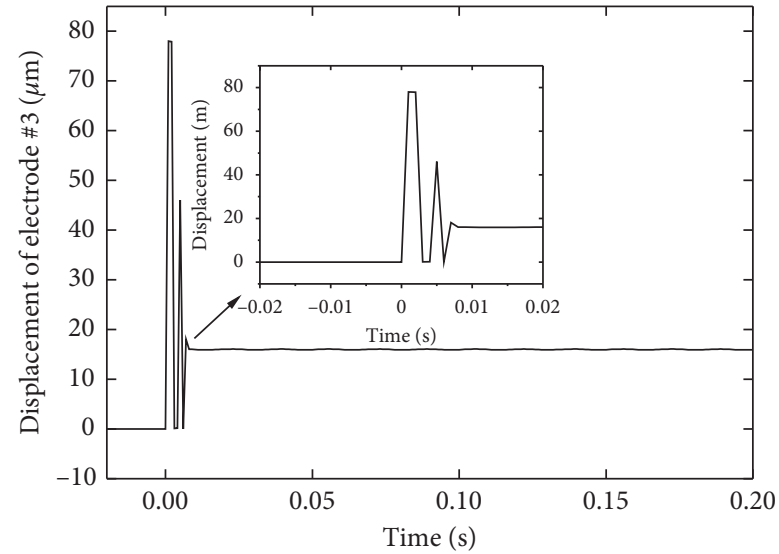

(f)

FIgURE 11: Suspension process of 4-inch silicon wafer.

position, the displacements of movable electrodes \#1, \#2, and \#3 were about 21,24 , and $16 \mu \mathrm{m}$, respectively. It can be concluded that the displacements of movable electrodes during stable suspension are controlled in the limited range without the occurrence of saturation of piezo actuators.

Compared to the simulation results presented in the previous section, small fluctuations occur when the suspended object is in its equilibrium position. This oscillation is recorded with an approximation of $0.30 \mu \mathrm{m}$. This oscillation is due to an error in the modeling of piezo actuator and mechanical amplifier when hysteresis of piezo actuator and nonlinearity of mechanical amplifiers are ignored. However, these oscillations of the object are very small, and they can be acceptable in contactless suspension process $[32,33]$. This model error also causes the difference in movements of movable electrodes between simulation and experimental results. Nevertheless, it is obvious that the simulated curves have a similar tendency as the experimental 
results and the good agreement between these results demonstrates the validity of the proposed method.

\section{Conclusion}

This paper presents the proximate time optimal control and stability analysis for the suspension system using a piezo actuator. The simulation and experiment results show that the suspension system using proximate time optimal control works well. This controller overcomes the piezo actuator saturation problem in the suspension system. The 4-inch silicon wafer was suspended fully at the reference gap $300 \mu \mathrm{m}$ with very small amplitude of vibrations. Since the voltage supplied to the piezo actuator must be a positive value, then the voltage supplied to the movable electrode needs to be smaller than the bias voltage which depends on the suspension gap. This system can be applied in equipment used in a spacious environment.

\section{Data Availability}

The data used to support the findings of this study are included within the article.

\section{Conflicts of Interest}

The authors declare that there are no conflicts of interest regarding the publication of this paper.

\section{Acknowledgments}

The authors are very grateful for the support received from the Ho Chi Minh City University of Food Industry and Ho Chi Minh City University of Technology for this project.

\section{References}

[1] E. H. Brandt, "Levitation in physics," Science, vol. 243, no. 4889 , pp. 349-355, 1989.

[2] H. W. Kneel, "The electric vacuum Gyro," Control Engineering, vol. 11, pp. 70-73, 1964.

[3] M. Ota, S. Andon, and H. Inoue, "Mag-lev semiconductor wafer transported for ultra-high vacuum environment," in Proceedings of Second International Symposium on Magnetic Bearings, pp. 109-114, Tokyo, Japan, July 1990.

[4] R. G. Ford and A. S. Koh, "Noncontact semiconductor wafer handling," in Proceedings of the Society of Manufacturing Engineers Annual Meeting on Semiconductor Manufacturing, pp. 1-10, Tempe, AZ, USA, July 1990.

[5] P. Paul-Francois, T. Ishikawa, G.-W. Lee et al., "Materials properties measurements and particle beam interactions studies using electrostatic levitation," Materials Science and Engineering: R: Reports, vol. 76, pp. 1-53, 2014.

[6] J. U. Jeon, J. Jin, and T. Higuchi, "Electrostatic suspension of 8-inch silicon wafer," in Proceedings of the Institute of Electrostatics, vol. 21, no. 2, pp. 62-68, Japan, 1997.

[7] J. Jin, T. C. Yih, T. Higuchi, and J. U. Jeon, "Direct electrostatic levitation and propulsion of silicon wafer," IEEE Trans. Industry Applications, vol. 34, no. 5, pp. 975-984, 1998.

[8] J. U. Jeon and T. Higuchi, "Electrostatic suspension of dielectrics," IEEE Transactions on Industrial Electronics, vol. 45, no. 6, pp. 938-946, 1998.
[9] J. U. Jeon and T. Higuchi, "Induction Motors with electrostatic suspension," J. Electrostatic, vol. 45, no. 2, pp. 157-173, 1998.

[10] J. Jin, T. Higuchi, and M. Kanemoto, "Electrostatic levitator for hard disk media," IEEE Transactions on Industrial Electronics, vol. 44, no. 2, pp. 234-239, 1997.

[11] J. Jin and T. Higuchi, "Direct electrostatic levitation and propulsion," IEEE Transactions on Industrial Electronics, vol. 44, no. 2, pp. 234-239, 1997.

[12] L. T. Truyen and U. P. Jeon, "Electrostatic suspension system using bang-bang time optimal control and stability analysis," in Proceedings of the 10th International conference on Control Automation Robotics and Vision, Hanoi, Vietnam, December 2008.

[13] J. U. Jeon and S. Lee, "Electrostatic suspension system of silicon wafer using relay feedback control," Journal of the Korean Society for Precision Engineering, vol. 22, no. 10, pp. 56-64, 2005.

[14] J. U. Jeon, B. W. Baik, H. M. Ngo, and K. Y. Park, "Electrostatic suspension system using time optimal control," Journal of the Korean Society for Precision Engineering, vol. 25, no. 3, pp. 63-72, 2008.

[15] L. T. Truyen and U. J. Jeon, "Stability analysis of time-optmally controlled electrostatic suspension system and suspension experiment in vacuum," Proceedings of the Institution of Mechanical Engineers Part C Journal of Mechanical Engineering Science, vol. 225, no. 1, pp. 88-100, 2010.

[16] T. W. Na, J. H. Choi, J. Y. Jung et al., "Compact piezoelectric tripod manipulator based on a reverse bridge-type amplification mechanism," Smart Materials and Structures, vol. 25, no. 9, Article ID 095028, 2016.

[17] C. Lin, Z. Shen, Z. Wu, and J. Yu, "Kinematic characteristic analysis of a micro-/nano positioning stage based on bridgetype amplifier," Sensors and Actuators A: Physical, vol. 271, pp. 230-242, 2018.

[18] P.-Z. Li, X.-D. Wang, Y.-X. Sui et al., "Piezoelectric actuated phase shifter based on external laser interferometer: design, control and experimental validation," Sensors, vol. 17, no. 4, p. 838, 2017.

[19] A. S. Bezryadina, D. C. Preece, J. C. Chen, and Z. Chen, "Optical disassembly of cellular clusters by tunable tug-of-war tweezers," Light: Science \& Applications, vol. 5, no. 10, Article ID e16158, 2016.

[20] M. Athans and P. L. Falb, Optimal Control-an Introduction to the Theory and its Applications, McGraw-Hill Book Company, New York, NY, USA, 1966.

[21] S.-T. Wu, "Time-optimal control and high-gain linear state feedback," International Journal of Control, vol. 72, no. 9, pp. 764-772, 1999.

[22] M. Kalyon, "Design of continuous time controllers having almost minimum time response," Journal of Dynamic Systems, Measurement, and Control, vol. 124, no. 2, pp. 252-260, 2002.

[23] G. Cheng and J.-g. Hu, "Robust proximate time-optimal servomechanism with speed constraint for rapid motion control," Robotics and Computer-Integrated Manufacturing, vol. 30, no. 4, pp. 379-388, 2014.

[24] G. F. Franklin, J. D. Powel, and M. Workman, Digital Control of Dynamic System, Addison Wesley Longman, Inc., Boston, MA, USA, 1998.

[25] D. E. Kirk, Optimal Control Theory-an Introduction, Prentice-Hall Inc., Englewood Cliffs, NJ, USA), 1970.

[26] L. Y. Pao and G. F. Franklin, "Proximate time optimal control of Third-order servomechanisms," IEEE Trans. Automatic Control, vol. 38, no. 4, pp. 560-580, 1993. 
[27] M. L. Workman, R. L. Kosut, and G. F. Franklin, "Adaptive proximate time optimal servomechanisms: discrete-time case," in Proceeding of the 26th Conference on Decision and Control, pp. 1548-1553, Los Angeles, CA, USA, 1987.

[28] http://www.nec-tokin.com.

[29] J. H. Kim, S. H. Kim, and Y. K. Kwak, "Development and optimization of 3-D bridge-type hinge mechanisms," Sensors and Actuator A: Physical, vol. 116, no. 3, pp. 530-538, 2004.

[30] S. B. Choi, "A magnification device for precesion mechanisms featuring piezoatuators and flexures hinges: design and experimental validation," Mechanism and Machine Theory, vol. 42, no. 9, pp. 1184-1198, 2007.

[31] S. T. Smith, Flexures-elements of Elastic Mechanisms, CRC Press, New York, NY, USA, 2000.

[32] L. Fridman, V. Strygin, and A. Polyakov, "Stabilization of oscillations amplitudes via relay delay control," in Proceeding of the 40th Conference on Decision and Control, pp. 37043709, Orlando, FL, USA, 2001.

[33] L. T. Truyen and U. P. Jeon, "Time delay effects on perfomance and stability of a low cost electrostatic suspension system," International Journal of Precision Engineering and Manufacturing, vol. 11, no. 4, pp. 549-557, 2010. 


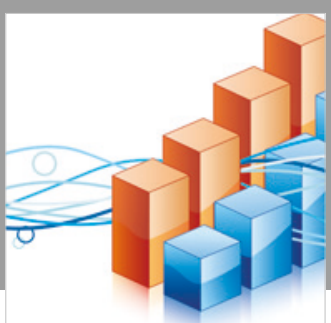

Advances in

Operations Research

\section{-n-m}
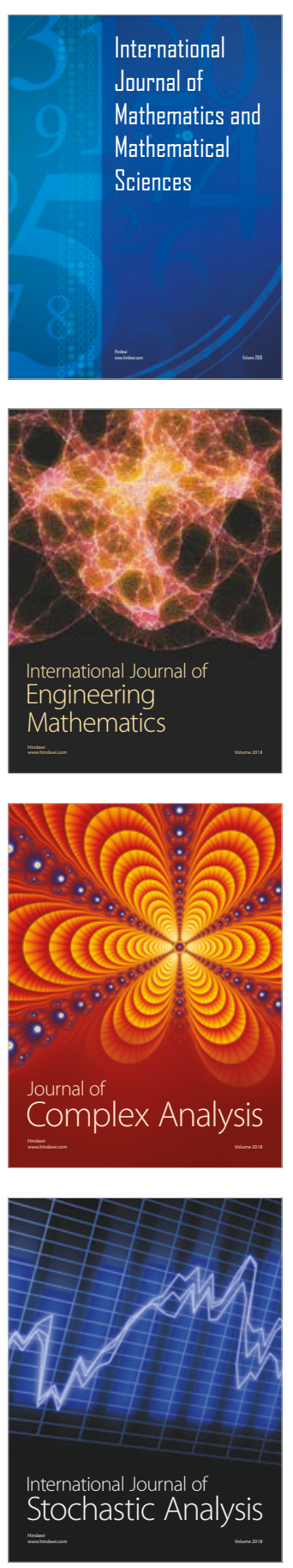
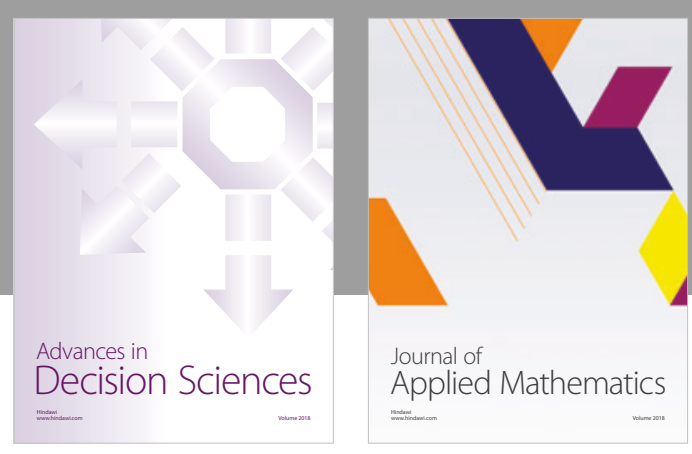

Journal of

Applied Mathematics
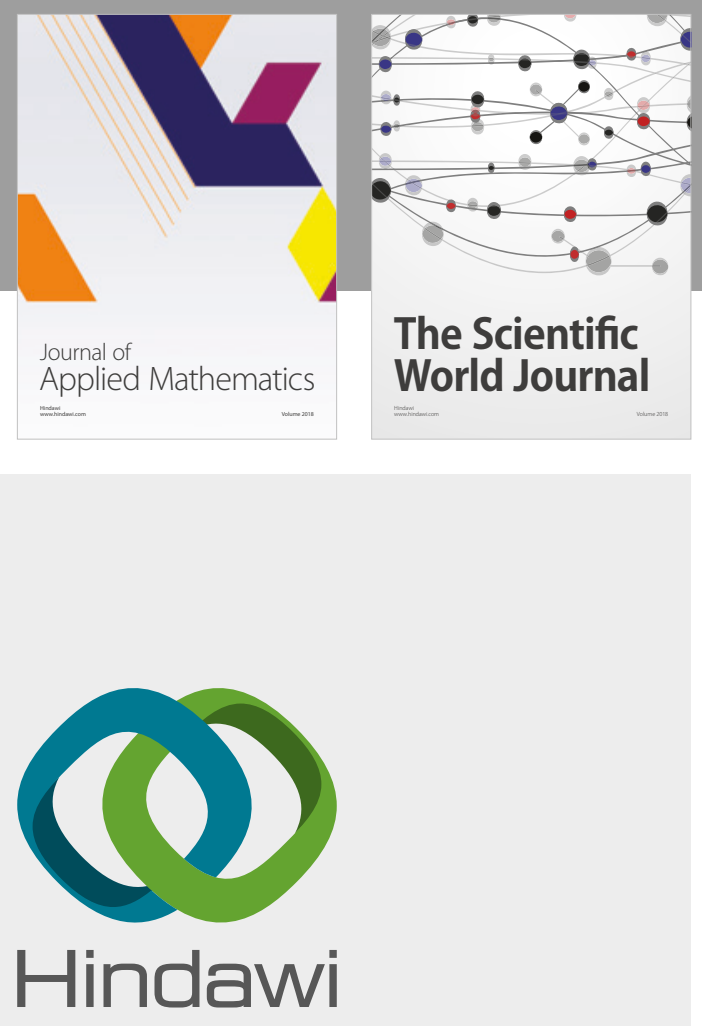

Submit your manuscripts at

www.hindawi.com

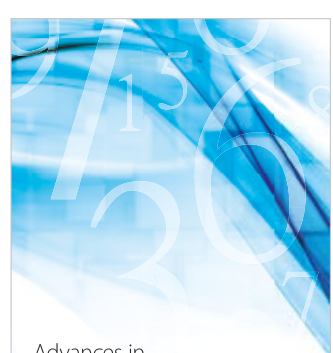

Advances in
Numerical Analysis
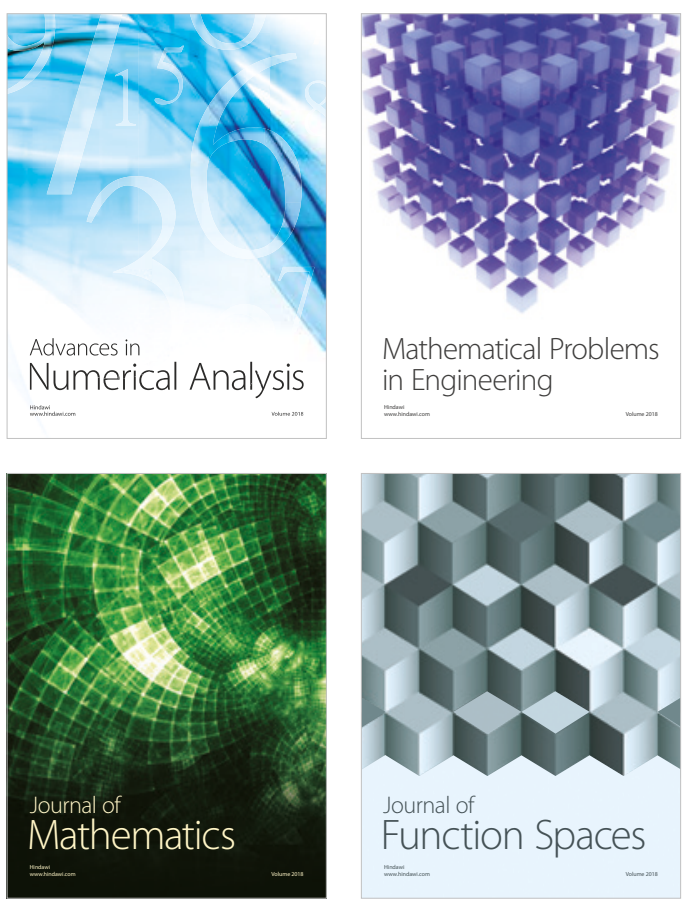

Mathematical Problems in Engineering

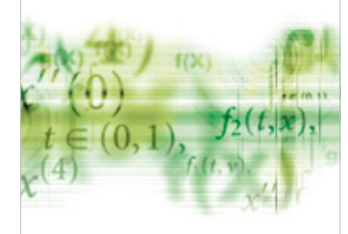

International Journal of

Differential Equations

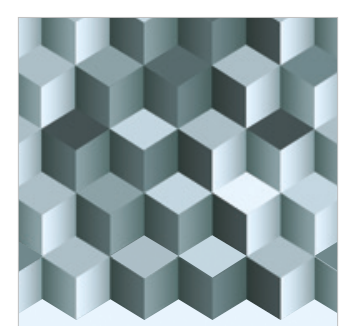

Journal of

Function Spaces

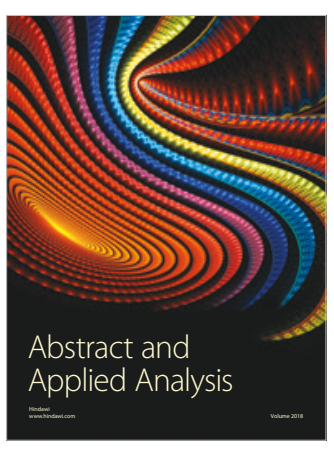

The Scientific

World Journal

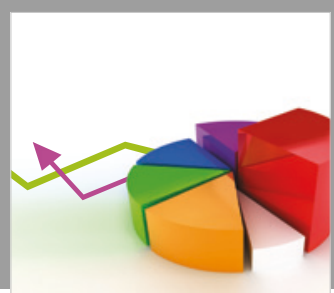

Journal of

Probability and Statistics
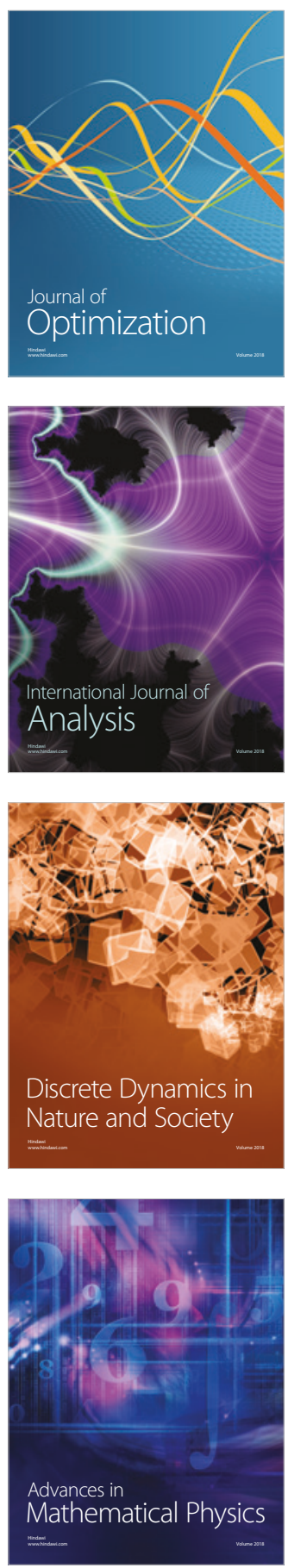